ISSN: 2602-8506

Vol. 3, N².1., p. 6-34, junio, 2019

Recibido: 05-04-2019 /Aceptado: 08-05-2019/ Publicado: 07-06-2019

\title{
La planificación tributaria como herramienta financiera para la optimización de los recursos empresariales
}

\section{Tax Planning as a Financial Tool to Optimize Business Resources}

Romel Patricio Mena Trujillo. ${ }^{1}$, Cecilia Ivonne Narváez Zurita. ${ }^{2}$ \& Juan Carlos Erazo Álvarez. ${ }^{3}$

DOI: https://doi.org/10.33262/visionariodigital.v3i2.1.540

\begin{abstract}
.
The improvement in the payment of taxes and the appropriate use of the benefits prescribed by the Organic Law of the Internal Tax Regime on the activities of natural and legal persons within business areas and services field have been set up as real optimization strategies of economic resources for the benefit of taxpayers. Consequently, the accurate and detailed assessment of the information relating to the commercial, financial and accounting situation of the taxpayer under the operating fiscal and legal framework allows to carry out assessments on alternatives proposed by the legislation to optimize the tax load in some cases, and the exemption from income tax payment in others.

Against this background, this research aims to design a tax planning for the Corporation of Construction and Planning Macas Macascorp S.A. that is located in the city of Macas, Ecuador. First, as a research strategy, the review of the specialized literature was conducted to point out the variables of the study. Subsequently, the diagnosis of the company supported by surveys and bibliographic review to establish the business structures and its tax risk were carried out. Finally, strategies were designed based on the identified tax benefit, and its impact on financial statements was valued through the Dupont System.
\end{abstract}

\footnotetext{
${ }^{1}$ Universidad Católica de Cuenca, Posgradista en Contabilidad y Auditoría, Cuenca, Ecuador, rpmenat582@psg.ucacue.edu.ec

${ }^{2}$ Universidad Católica de Cuenca, Posgrados, Cuenca, Ecuador. inarvaez@ucacue.edu.ec

${ }^{3}$ Universidad Católica de Cuenca, Posgrados, Cuenca, Ecuador. jcerazo@ucacue.edu.ec
} 
Keywords: Tax planning, taxes, tax load, tax evasion, tax avoidance

\section{Resumen}

El perfeccionamiento en el pago de impuestos y el uso apropiado de los beneficios atribuidos en la Ley Orgánica de Régimen Tributario Interno (LORTI) en actividades de personas naturales y jurídicas, en las áreas comerciales y de servicios se han constituido en la actualidad en verdaderas estrategias de optimización de recursos económicos en beneficio de los contribuyentes. Por consiguiente, la evaluación oportuna y detallada de la información relacionada con la situación comercial, financiera y contable del contribuyente en el marco del régimen fiscal y legal vigente, permite realizar valoraciones sobre las alternativas que prevé la legislación para optimizar la carga fiscal en unos casos y en otros lograr la exoneración el pago del impuesto a la renta.

En este contexto, la presente investigación tiene por finalidad diseñar una planificación tributaria para la empresa Constructora y Planificación Macas Macascorp S.A., localizada en la ciudad de Macas, Ecuador. Para tal efecto se aplicó como estrategia de investigación la revisión de literatura especializada para fundamentar las variables de estudio, posteriormente se realizó un diagnóstico tributario de la compañía para establecer la estructura de negocio y el riesgo tributario apoyados en cuestionarios y revisión documental, finalmente se diseñaron las estrategias basadas en los beneficios fiscales identificados y se avaluó su impacto en los estados financieros mediante el sistema Dupont.

Palabras claves: planificación tributaria, carga tributaria, impuesto a la renta.

\section{Introducción.}

A nivel internacional se considera a la industria de la construcción como uno de los más importantes detonantes del crecimiento económico de un país, no solamente por las empresas que directamente se dedican a esta actividad, sino también, por el efecto multiplicador que genera en la contratación de mano de obra y de otras industrias que indirectamente intervienen (Instituto de Desarrollo Industrial, Tecnológico y de Servicio, 2009). 
Según el Instituto Nacional de Estadistica y Censos - INEC (2012), en el Ecuador existen cerca de quince mil empresas directa o indirectamente relacionadas con actividades de construcción, de éstas, aproximadamente unas once mil se dedican a proveer los insumos necesarios para esa actividad, tales como: la metal mecánica, hierro, acero, cemento entre otros. Es así que, un 75\% de las empresas están inmersas en el efecto multiplicativo de esta actividad y si se considera la mano de obra se verifica la importancia del sector de la construcción en la economía ecuatoriana.

Al respecto, la Dirección Nacional de Investigación y Estudios (2016) señala que en la amazonia ecuatoriana existen trecientas doce empresas constructoras, de las cuales el $95 \%$ son micro o pequeñas empresas con ventas anuales menores a un millón de dolares, y el cinco por ciento restante, corresponde a empresas con ventas mayores a un millón de dólares, en las cuales trabajan directamente dos mil personas, siendo el sector de la construcción, la mayor fuente generadora de de empleo privado en la región oriental.

No obstante, la caída del precio del petróleo a finales del 2015 y durante el 2016, conjuntamente con una falta de previsión sobre este ingreso importante para la economía nacional, obligó al Estado a restructurar su presupuesto y priorizar el uso del dinero, afectando directamente al sector constructor, el mismo que sobrellevó un rezago de casi tres años consecutivos, periodo durante el cual, se dio la suspensión del financiamiento de proyectos públicos, y la generación de pagos por parte del Estado sobre proyectos ejecutados. Esta crisis económica sumada a las cambiantes políticas tributarias que persiguen un mayor control estatal a fin de evitar el uso abusivo de las deducciones, las rebajas, diferimientos y otros beneficios establecidos legalmente (Sánchez y Hablich, 2018), han conllevado a que las empresas busquen optimizar sus recursos mediante la aplicación de estrategias de planificación tributaria. 
En este sentido, la administración tributaria del Ecuador (Servicio de Rentas Internas), con la finalidad de reducir tanto la evasión ${ }^{4}$ como la elusión ${ }^{5}$ de impuestos ha obligado al sector empresarial a aplicar procedimientos que garanticen el cumplimiento oportuno de sus obligaciones tributarias, bajo contextos de veracidad, confiabilidad e integridad.

Por lo antes expuesto, en el presente estudio se ha seleccionado al sector de la construcción, y dentro de éste, a la constructora Macascorp S.A., constituida en la provincia amazónica de Morona Santiago, con domicilio tributario en la ciudad de Macas, por ser una mediana empresa generadora de ingresos importantes, y principalmente por el impacto de sostenibilidad económica de la región amazónica.

\section{La planificación tributaria en Sudamérica}

Venezuela es uno de los países pioneros en la utilización de la planificación tributaria en América del Sur, hace una década atrás, inició la avidez del Estado por la obtención de recursos económicos y el incremento de la carga tributaria también conocida como presión fiscal, causando que los empresarios implementen sistemas técnicos que les permitan pagar menos impuestos. Al respecto, Hidalgo (2009) menciona generalidades de la planificación tributaria como son los conceptos, políticas y las metas a conseguir, además hace un importante análisis sobre las distintas motivaciones que tuvo la empresa privada venezolana para buscar estrategias que les permita mantenerse de pie ante la creciente carga tributaria que es el principal motivador de la implementación de sistemas de optimización de impuestos o planificaciones tributarias dentro de las empresas.

En la figura 1 se observa la presión fiscal o carga tributaria de Ecuador y los demás países de Sudamérica, con excepción de Venezuela y Bolivia que por su nivel de impuestos a las exportaciones no permite comparar su recaudación impositiva con los demás países de la región.

\footnotetext{
${ }^{4}$ Rivas y Vergara (2002) conceptualizan a la evasión tributaria como una conducta dolosa o maliciosa para disminuir parcial o totalmente el monto de los tributos a pagar, violentando u omitiendo las dispociciones legales, dejando de lado la ética y los valores en los que se fundamentan las empresas.

${ }^{5}$ La elusión fiscal es una modalidad legal y planificada de resistencia fiscal y en ningún caso cae en lo inmoral, es un principio básico de los Estados que respetan la libertad (Tipker, 2002), adicionalmente.
} 
ISSN: 2602-8506

Vol. 3, N².1., p. 6-34, junio, 2019

Ecuador pasa de último lugar recaudador en el 2004, a ser el primero en 2015, este análisis sin considerar a: Argentina, Brasil y Uruguay, países que están muy por encima del promedio de la región. Cabe mencionar que Ecuador en comparación con sus dos principales competidores: Colombia y Perú, ejerce una mayor carga tributaria siendo un punto porcentual mayor al primero y cuatro puntos que el segundo. Por tanto, desde el punto de vista interno, en la última década la carga tributaria en Ecuador se incrementó en un 50\% pasando del 13.5\% en el 2005, al $21.7 \%$ en el 2015, mientras que; comparativamente pasó de ocupar el penúltimo lugar, al cuarto en el mismo período de tiempo analizado, justificando que las empresas pretendan optimizar sus impuestos a través de herramientas como la planificación tributaria.

Figura 1. Presión Fiscal en América del Sur

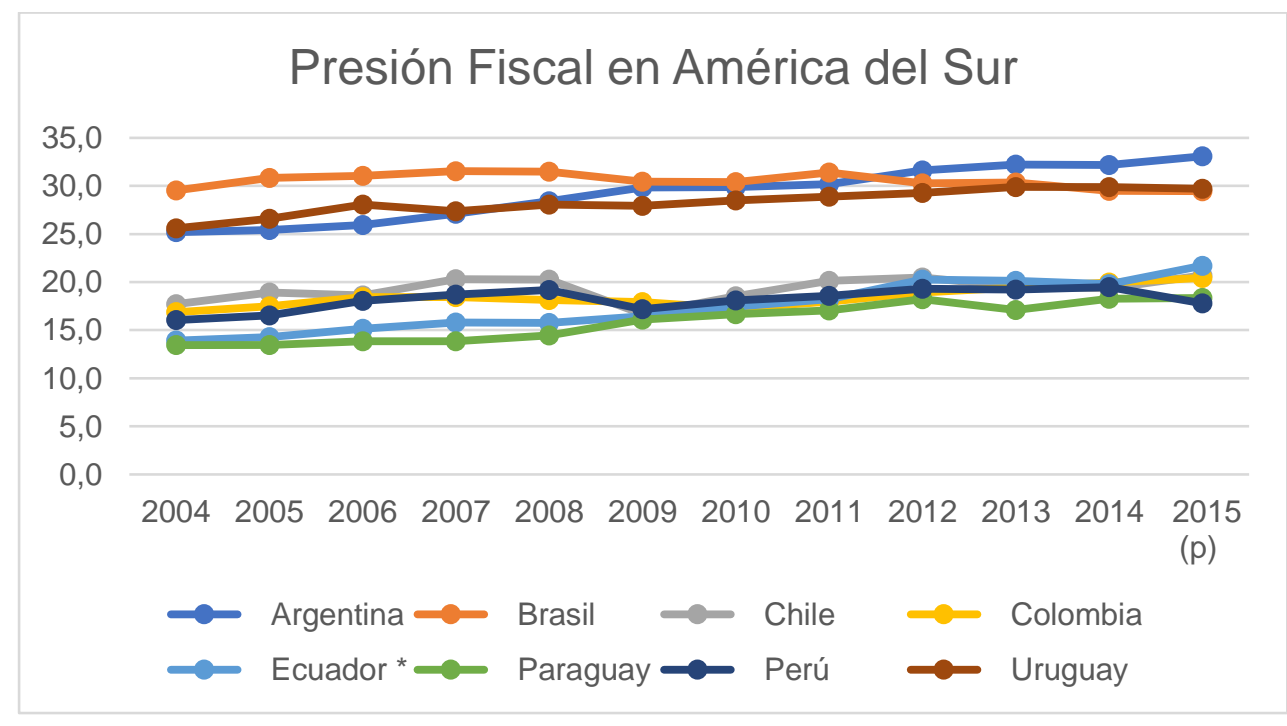

Fuente: Adaptado de Visores Tributarios (SRI, 2018)

\section{La planificación tributaria como herramienta financiera}

Acevedo (1998) señala que la planificación tributaria (PT) es una herramienta de la administración cuya finalidad es la determinación justa del pago de impuestos, conociendo las consecuencias fiscales de las decisiones de las personas naturales o jurídicas. Paralelamente, Cusgüen (1998), desarrolló un concepto de PT amplio en el cual la describe como lineamientos apropiadamente alineados con la realidad de cada empresa que considera entre otras cosas: las estrategias, políticas 
y controles organizacionales, antecedentes y expectativas de la misma para que todo en conjunto sea una guía de trabajo para el personal especializado encargado del área tributaria.

Por otra parte, autores como Ruiz (1998), Rivas y Vergara (2002), conceptualizan a la PT apegada al tratamiento legal y no administrativo, señalando que se trata simplemente de un proceso en el cual se orientan las decisiones y acciones de las organizaciones a la par de las consecuencias impositivas que pudieran derribarse de las mismas, procurando aminorar el pago de sus obligaciones sin oponerse de ninguna manera al ordenamiento tributario. Así también, Galárraga (2002), y Cámara (2005), conceptualizan a la planeación como un proceso definido en el cual se delimitan actividades fundamentadas, con la finalidad de conseguir objetivos específicos; es decir que es una consecución de acciones determinadas lógicamente para alcanzar una meta, además los autores concuerdan que la consecución del objetivo será supeditada a la transparencia y racionalidad de las decisiones tomadas.

Posteriormente, Parra (2007), desarrolla un concepto de PT combinando las perspectivas antes mencionadas y definiéndola como controles de las distintas obligaciones ya sean impuestos, tasas y/o contribuciones que genera la empresa, además; advierte que se debe deducir de los ingresos totales los impuestos y también la utilidad neta, pago de acreedores y el crecimiento de la empresa asegurando además dividendos justos a los accionistas previniendo riesgos tributarios que puedan conllevar la toma de decisiones. Una década más tarde, Osar y Ramos ( 2017), señalan que para que una organización en sentido rentista obtenga su óptimo rendimiento financiero necesita ordenar sus recursos administrativos siendo los impuestos parte de ellos, por lo que, unos de los objetivos de la organización es considerar mecanismos de procedimientos fiscales y de control tributario, anticipando el valor de impuesto a pagar provisionándolas durante el período contable que generalmente es de un año.

De los conceptos planteados se considera los aportes de Cusguien (1998) y Parra (2007) ya que son de mayor utilidad para el estudio propuesto, debido a que asumen a la PT no solo como herramienta para el pago de impuestos sino más bien como una herramienta de carácter financiero o gerencial, la misma que se articula a la misión y visión de la empresa y genera o delimita las decisiones a tomar durante un período para optimizar los recursos, repartir utilidades y pagar los 
impuestos que deban generarse previamente planificados, considerando los riesgos de carácter tributario que se podrían acarrear.

En los párrafos anteriores, se conceptualizó a la PT como un instrumento cuya finalidad es disminuir los valores a pagar por el rubro de impuestos sin que se cometa ninguna infracción de tipo tributaria y que además no se interrumpa en el desarrollo de la actividad económica de la organización. De la misma manera pretende cambiar los lineamientos administrativos realizados de forma artesanal por métodos sofisticados que tengan un panorama financiero claro al momento de tomar decisiones (Cusgüen, 1998). En este sentido, para que la PT sea considerada como una herramienta financiera debe propender a la optimización de los recursos destinados para cumplir las obligaciones de carácter tributario, prever los montos a pagar y plantear estrategia para que los recursos que se economicen fortalezcan las finanzas de la organización.

Ahora bien, si se analiza las distintas transacciones económicas que realiza la organización sin que se consideren los impuestos implícitos en cada una de ellas, se está sobreestimando los ingresos, mientras que se sub estiman los egresos, teniendo como consecuencia final una sobre estimación de las utilidades y expectativas de dividendos de los socios (Cusgüen, 1998). En tal caso, la PT como herramienta financiera considera los siguientes aspectos: permite disminuir legalmente los recursos monetarios destinados al pago de impuestos sin que se interfiera con las actividades normales de la organización, además actualiza los métodos administrativos según su influencia en los tributos y propende optimizar coordinadamente los esfuerzos invertidos para el cumplimiento de los objetivos, tanto tributarios como organizacionales de manera eficiente y eficaz.

En el mismo escenario, para poder anticipar la cifra del impuesto a pagar, la planificación tributaria tiene como uno de sus objetivos fundamentales calcular la distribución de la carga impositiva durante un período que generalmente es de un año (periodo fiscal), con la finalidad de obtener el recurso monetario y cumplir con los montos y plazos previsto para el pago y así lograr la extinción de la obligación tributaria apegados a las normas jurídicas y a la responsabilidad con la sociedad. 
Como se denota, la planificación tributaria procura optimizar los recursos de la empresa y es por ello que a más de considerar la parte tributaria se necesita eficiencia y eficacia en el manejo de los recursos, por lo que herramientas de análisis financieros como las razones o ratios financieros son necesarios para el efecto; cabe recalcar que ningún ratio por separado permite evaluar el desempeño de carácter financiero (López, 2017). No obstante, el sistema Dupont es planteado como una herramienta decisional ya que mediante la conjunción de varias razones financieras concluye en la medición de la optimización en la utilización de los recursos empresariales, además proporciona la información pertinente para evaluar el grado de control en los costos y gastos erogados para la obtención de los ingresos. Autores como García (2014), y Dupont (2018), concuerdan con lo señalado y añaden la importancia de vincular dinámicamente la información de los Estados Financieros, siendo la fórmula así:

Figura 2. Representación Gráfica de la fórmula de Dupont

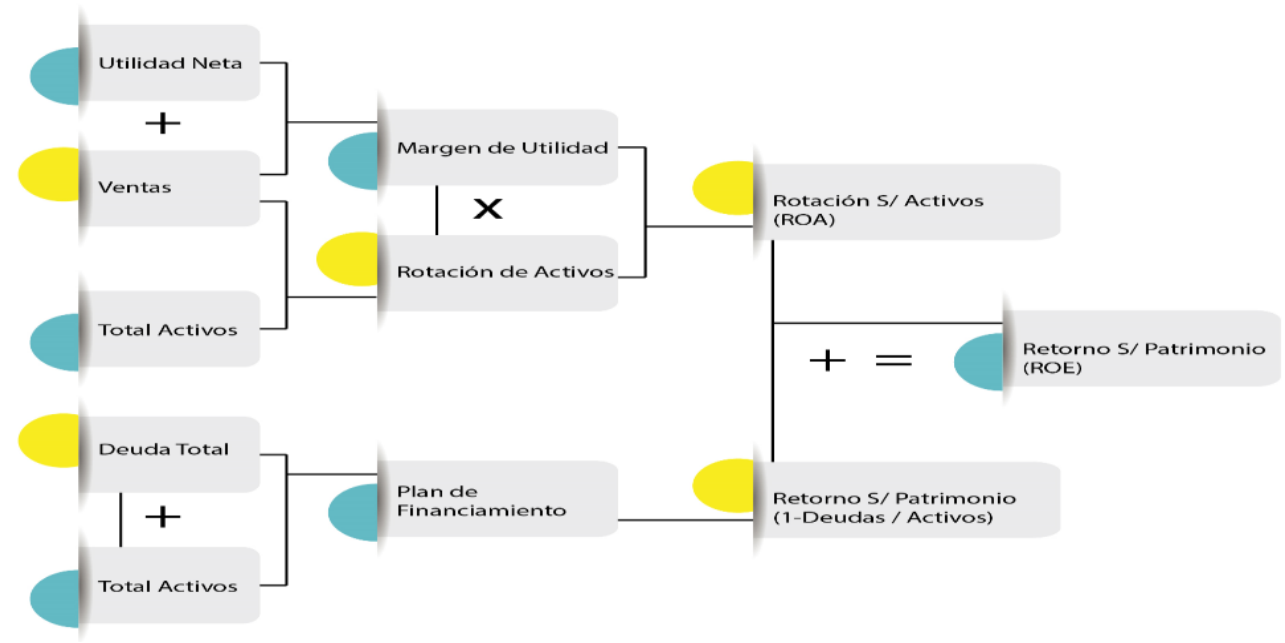

Fuente: García (2014).

El sistema Dupont interrelaciona los estados financieros a través del rendimiento sobre activos (ROA), del retorno sobre el patrimonio (ROE) y el margen de apalancamiento financiero (MAF). Es decir, primeramente, relaciona el margen de utilidad con las ventas y la inversión en activos para luego continuar con la deuda contraída para financiar tales activos tanto como deuda con terceros como la contraída con los socios, terminando el análisis en el beneficio obtenido por los 
socios comparado con su inversión, permitiendo así una evaluación integral de las decisiones tomadas por la administración en un período determinado.

\section{Principios y objetivos de la planificación tributaria}

Los principios y objetivos de la PT se basan en la libertad que se tiene en derecho privado de hacer todo lo que no esté prohibido y para la legalidad del cobro de los tributos previamente debe existir un vínculo jurídico o normativo, los principios señalan el camino a seguir, los objetivos muestran el propósito y los resultados esperados por los cuales se están realizando la planificación, (Rivas y Vergara, 2002).

Figura 3. Principios de la Planificación Tributaria

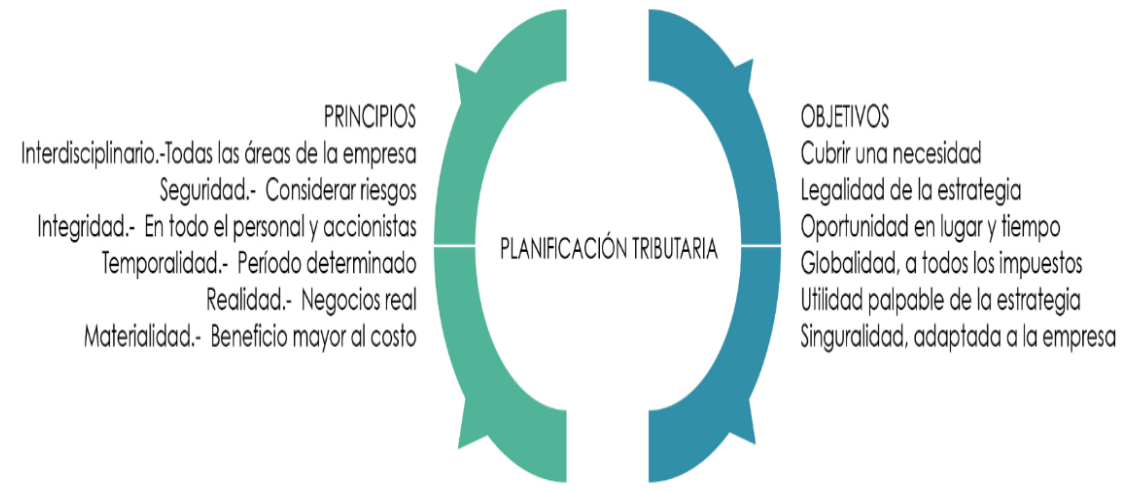

Fuente. Adaptado de Rivas y Vergara (2002).

Para una adecuada planificación tributaria se deben considerar elementos básicos y fundamentales dentro de los cuales se encuentran los siguientes: que sea un negocio existente, que la forma jurídica de la organización se maneje como unidad económica entre propietarios y empresa, que considere todos los tributos que se relacionen con el giro del negocio, análisis del entorno y su efecto en la organización y por último que tenga una perspectiva global capaz de acoplar todas las estructuras antes señaladas .

Cada elemento según Parra (2007) se encamina a la consecución de las metas que persigue la PT, las mismas que consisten:

- Elegir estratégicamente la forma jurídica y administrativa de la organización. 
- Evitar la realización de hechos económicos gravados innecesariamente.

- Utilizar de forma correcta los incentivos tributarios otorgados por el Estado, y.

- Equilibrar el costo beneficio de la inversión y los impuestos.

Las fases o etapas que conllevan una PT, deben como todo proceso seguir un ordenamiento lógico para poder obtener inequívocamente los resultados planeados, por lo que, a continuación, se plantea las principales etapas que cuenta una PT a especie de hoja de ruta a seguir para elaborar el proyecto de investigación.

Figura 4. Etapas de la Planificación Tributaria.

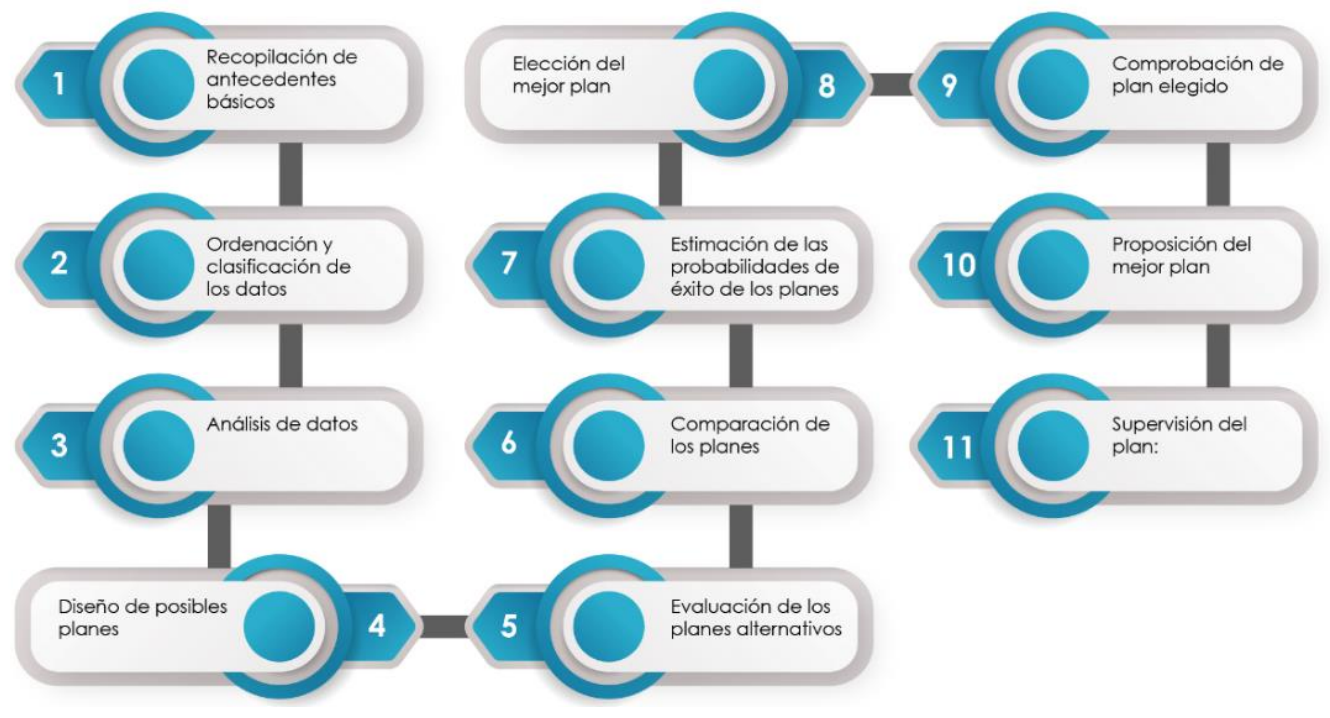

Fuente: Adaptado de Rivas y Vergara (2002)

Cada empresa es un ente distinto, por lo que los métodos aplicados serán también diferentes y ajustados a las necesidades de las mismas, sin embargo, para que se pueda lograr la consecución de los objetivos planteados, los métodos deben seguir un ordenamiento lógico como son los procesos, es decir, que exista un vínculo entre métodos y procedimientos, conjugando directrices y principios, obteniendo como resultado un modelo único para cada empresa que delimite las decisiones tomadas de carácter tributario (Rivas y Vergara, 2002). 


\section{Metodología}

En la presente investigación se utilizó un enfoque cuali - cuantitativo, en virtud que se analizaron características específicas e individuales de las variables de estudio, así como también, se utilizaron herramientas estadísticas para la recolección y análisis de datos que sirvieron como base para el diagnóstico tributario en la empresa Constructora y Planificación Macas Macascorp S.A. En cuanto al alcance, la investigación fue descriptiva - explicativa, ya que, se describieron la planificación tributaria y sus componentes en el estado del arte y se caracterizó la carga fiscal de la unidad de análisis, mediante el levantamiento de datos en solo momento del tiempo.

Los métodos utilizados fueron el inductivo - deductivo, aplicado en dos etapas, la primera se refiere al conocimiento general teórico de las variables en estudio para luego determinar sus dimensiones particulares. El método histórico - lógico, se utilizó en el análisis de los antecedentes, evolución y tendencias de la planificación tributaria y finalmente el método analítico - sintético permitió la descomposición de las variables a fin de estudiar cada uno de sus componentes para luego integrarlos en un todo. Las técnicas aplicadas fueron la encuesta, la revisión documental y la observación directa con la finalidad de efectivizar los métodos investigativos y el tratamiento de la información desde la recopilación hasta su análisis.

A modo de ejemplo, se presenta el examen de evaluación del riesgo tributario, considerando los distintos parámetros de la normativa tributaria vigente.

Tabla 1. Examen del riesgo tributario 
Macascorp S.A.

Examen: Evaluación del riesgo tributario de la empresa

Periodo: entre el 1 de enero de 2018 y el 31 de diciembre de 2018



Fuente: Elaboración propia

El diagnóstico empresarial se basa en conocimientos previos de la situación o problema que atraviesa la organización, evaluando los resultados para plantear una propuesta que responda a las 
necesidades aquí determinadas (Guizar, 2013). Por tanto, la información recopilada mediante las distintas técnicas estadísticas tuvo como objetivo presentar resultados de manera ordenada y sistematizada del conocimiento general que tiene el personal administrativo de la empresa y la normativa tributaria, así como, evaluar el riesgo tributario en el cual se desenvuelven las actividades empresariales, evaluando de esta manera la situación empresarial inicial y fijando las principales necesidades. Entre los resultados más relevantes de la fase de evaluación, se obtuvieron los siguientes:

- La empresa cuenta con una misión, visión y valores. De estos elementos se puede evidenciar que los trabajadores se encuentran familiarizados y conocen la misión y visión de la empresa, lo que potencia su trabajo con una proyección a mediano y largo plazo.

- Un alto porcentaje de los trabajadores conocen los valores que guían la conducta cotidiana de la empresa, para la consecución de la visión e identidad corporativa.

- En cuanto a los procesos contables y tributarios, estos se encuentran establecidos en la empresa, sin embargo, no se organizan y delimitan con frecuencia las actualizaciones o reformas tributarias.

- En lo que respecta al cumplimiento de la normativa, la empresa cumple formalmente con los reglamentos de comprobantes de venta y retenciones, y emisión de comprobantes complementarios.

- La brecha de legalidad considera que la empresa está debidamente constituida, tiene su registro único de contribuyente (RUC) y las actividades económicas que realiza están enmarcadas en su objeto social (construcción de obras civiles y el alquiler de maquinaria).

- La empresa está inmersa en el sistema de facturación electrónica, por consiguiente, sus comprobantes de venta y retención son autorizados en el momento de su emisión y es de conocimiento del SRI en tiempo real las transacciones que realiza.

- De la evaluación de la brecha de cumplimiento se determinó el retraso en la presentación y pago de IVA, mientras que el impuesto a la renta fue declarado y cancelado en los tiempos pertientes 
- Se observaró sanciones pecuniarias por los incumplimientosde la presentación y pago del IVA en tres de los doce meses del año 2018.

- De la evaluación de la brecha de veracidad se estableció que existe un fuerte control interno, por lo que, los procesos de validación de la documentación identifican y minimizan riesgos en la información, en este sentido, las distintas auditorías internas y externas por auditores calificados han confirmado la calidad de la información presentada.

- Finalmente, de las auditorias financieras e informes de cumplimiento externos realizadas a la organización, se verificó que todas fueron sin salvedad, mostrando una fortaleza en la calidad de la información interna. Sin embargo, de la evaluación del riesgo tributario que es presentada en su totalidad en la tabla 1 , se desprende la valoración del nivel de confianza bajo, causado principalmente por la inoportuna presentación y pago del IVA y la recepción de sanciones por la misma causa, en consecuencia, un riesgo tributario alto.

\section{Resultados}

Por encontrarse la empresa objeto de estudio en un nivel de confianza bajo y en un riesgo tributario alto, esta podrá contemplar la planificación tributaria propuesta para el año 2019, considerando, que además de los elementos financieros, las deducciones, las rebajas, y los diferimientos, se tuvo en cuenta los beneficios por localizarse en zona deprimida.

\section{Componentes de la planificación tributaria propuesta:}

El primer componente de la propuesta es el conocimiento preliminar de la empresa detallando los datos generales, así como: la misión, visión y valores de la misma; el siguiente componente aborda las metas y principios de planificación tributaria aplicados, seguidamente se realiza un análisis de los incentivos tributarios del sector y el cumplimiento de las obligaciones impositivas, para finalmente medir el impacto de la propuesta reflejando en los estados financieros su utilidad según comparabilidad del ahorro tributario y el cálculo del índice de Dupont planteado.

La estructura organizativa de Macascorp S.A. es clásicamente piramidal, en la cual la junta general de accionistas es la máxima autoridad decisional de la empresa seguida del representante legal que 
ISSN: 2602-8506

Vol. 3, N².1., p. 6-34, junio, 2019

www.visionariodigital.org

a la vez es el gerente general. Las tareas que desarrolla el equipo humano están plenamente establecidas en un sistema de control interno, permitiendo el desarrollo grupal como individual de cada miembro de la empresa.

Figura 5. Modelo de planificación tributaria

\section{MODELO DE PLANIFICACIÓN TRIBUTARIA}

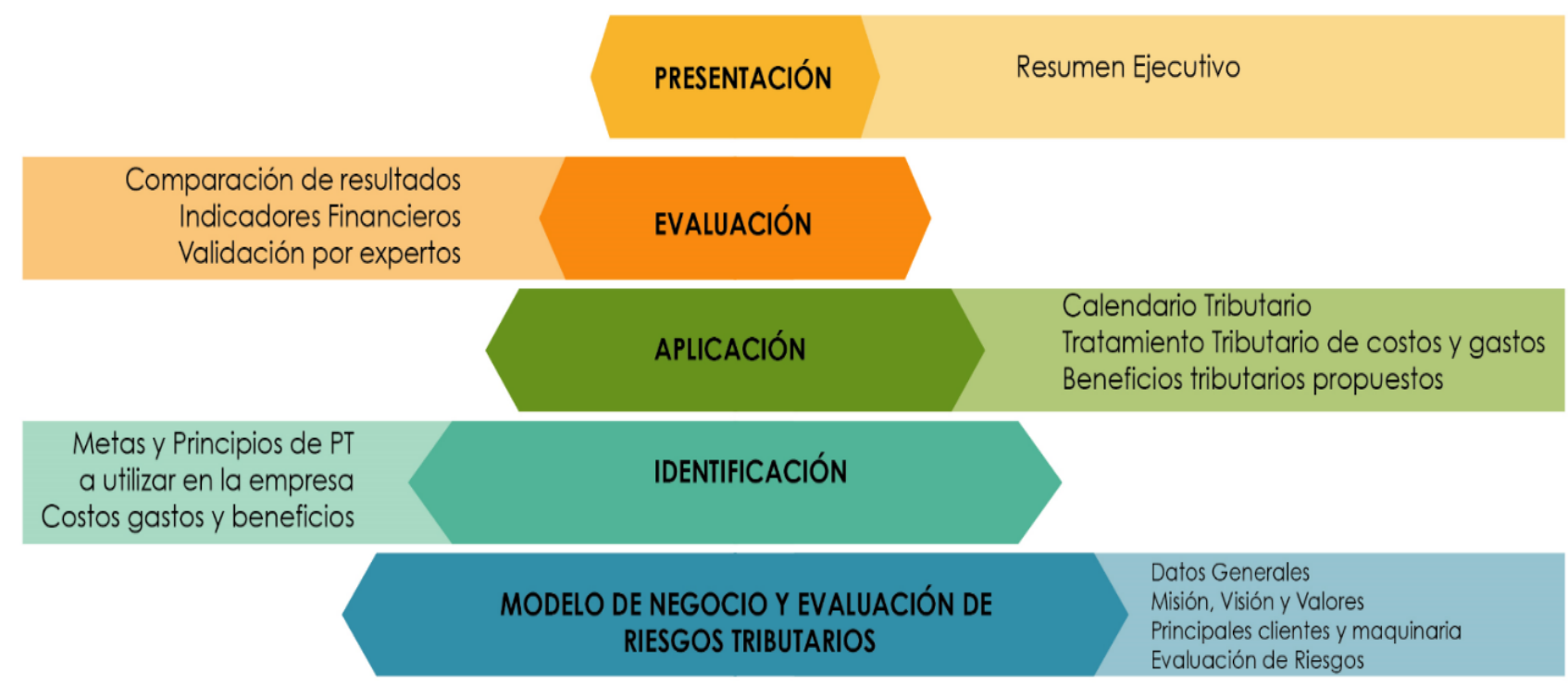

Fuente: Elaboración propia

Tabla 2. Datos Generales de la Empresa 
ISSN: 2602-8506

Vol. 3, N².1., p. 6-34, junio, 2019

\begin{tabular}{ll}
\hline Tipo de sociedad: & Sociedad Anónima \\
\hline Organismo regulador: & Superintendencia de Compañías \\
\hline Número de RUC: & 1490808414001 \\
\hline Razón social: & Constructora y Planificación Macas Macascorp S.A. \\
\hline Clase de contribuyente: & Sociedad \\
\hline Actividad económica principal: & Planificación, asesoramiento y construcción de obra civil \\
\hline Actividades económicas & Alquiler de maquinaria destinada para dicho fin \\
secundarias: & \\
\hline Dirección del domicilio tributario: & Av. 29 de Mayo s/n y Modesta Rivadeneira \\
\hline Medios de contacto: & 072322083 \\
\hline
\end{tabular}

Fuente: Adaptado del acta de constitución de la empresa

Principales maquinarias: Para realizar a cabalidad su actividad económica, la empresa cuenta con equipo caminero que a continuación se detalla:

Tabla 3. Vehículos y equipo caminero de la empresa

\begin{tabular}{|rlrr}
\hline \multicolumn{1}{|c}{ Fecha } & \multicolumn{1}{c}{ Descripción } & \multicolumn{1}{c}{ Factura } & \multicolumn{1}{c}{ Valor } \\
\hline 1-oct.-13 & Mercedes Benz 19-24 & Contrato Compra-Venta & $6.000,00$ \\
\hline 1-ene.-15 & Rodeo 4 X 4 DLX T/M A/C 2.6 L & Contrato Compra-Venta & $6.814,22$ \\
\hline 14-abr.-15 & Mercedes Benz, modelo DB2644 & Contrato Compra-Venta & $50.000,00$ \\
\hline 24-sep.-15 & Excavadora oruga, 7.1-3-000502, DOOSAN & 1005 & $110.000,00$ \\
\hline 14-may.-15 & Volqueta Mercedes Benz, modelo LK 1924 & Contrato Compra-Venta & 500,00 \\
\hline 16-sep.-15 & Volqueta Mercedes Benz modelo 2632 & Contrato Compra-Venta & $24.000,00$ \\
\hline 2-oct.-15 & Tráiler Mercedes Benz modelo 2636 & Contrato Compra-Venta & $45.000,00$ \\
\hline & \multicolumn{2}{c}{ Total Vehículos y Equipo Caminero } & $\mathbf{2 4 2 . 3 1 4 , 2 2}$ \\
\hline
\end{tabular}

Fuente: Adaptado de Mayores de Activos Fijos de la empresa 
Planificación tributaria a través de los ingresos: Macascorp S.A. obtiene sus ingresos directamente de los contratos que se firman con el Estado, ya que su giro comercial se basa y especializa en la construcción de carreteras pavimentadas o apertura de caminos en la provincia de Morona Santiago, por lo que sus ingresos en su totalidad son gravados.

Planificación tributaria a través de costos y gastos: Los gastos no deducibles se centran específicamente en la depreciación del equipo caminero, que al no contar con un comprobante de venta es considerado como no deducible al momento de generar el gasto por el uso del mismo. Estos egresos representan un incremento del $25 \%$ por impuesto a la renta. El incremento de impuestos genera una disminución de la utilidad en los estados financieros.

Tratamiento tributario de Costos y Gastos: Para la empresa en estudio se considera la depreciación del equipo caminero como gasto no deducible debido a que se adquirió con contratos y no con factura. No obstante, para el efecto de deducibilidad de las depreciaciones, en la ley y el reglamento no especifica que sea necesario un comprobante de venta para solventar la compra, sino que cumpla con los criterios para considerarlo como activo y su pago se realice empleando el sistema financiero, pudiendo ser considerada totalmente como deducible (Ver tabla 4).

Tabla 4. Depreciación propuesta

\begin{tabular}{|l|c|c|c|}
\hline Concepto & Valor & Depreciación & Saldo en Libros \\
\hline Muebles y Enseres & 787,13 & 78,71 & 708,42 \\
\hline Maquinarias menores & 510,83 & 170,28 & 340,55 \\
\hline Equipo Caminero & $242.314,22$ & $48.462,84$ & $193.851,38$ \\
\hline Equipo de Computo & 599,76 & 199,92 & 399,84 \\
\hline Edificaciones & $40.713,54$ & $2.035,68$ & $38.677,86$ \\
\hline Total depreciación & & $50.947,43$ & \\
\hline No deducible & & - & \\
\hline
\end{tabular}

Fuente: Adaptado de Mayores de Activos Fijos de la empresa

Beneficios tributarios propuestos: En base al análisis realizado, se propone la utilización de beneficios tributarios como la disminución de gastos no deducibles, el incremento neto de empleados, el reconocimiento de ingresos no planillados y reinversión de utilidades. De esto podemos proponer los siguientes puntos: 
ISSN: 2602-8506

Vol. 3, N².1., p. 6-34, junio, 2019

Tabla 5. Beneficios tributarios propuestos

\begin{tabular}{|c|c|c|c|}
\hline Incentivo A: & Por Concepto de: & Base Legal & Condiciones Internas \\
\hline \multirow{3}{*}{$\frac{\mathfrak{0}}{\frac{0}{\omega}}$} & $\begin{array}{l}\text { Los rendimientos y beneficios obtenidos por } \\
\text { personas naturales y sociedades, residentes o } \\
\text { no en el país, por depósitos a plazo fijo en } \\
\text { instituciones financieras del país }\end{array}$ & LRTI - 9.15 & $\begin{array}{l}\text { En obras mayores a un año } \\
\text { y en la parte del anticipo a } \\
\text { no ser utilizado }\end{array}$ \\
\hline & $\begin{array}{l}\text { Intereses por préstamos a trabajadores para } \\
\text { que adquieran acciones o participaciones de la } \\
\text { sociedad empleadora. }\end{array}$ & LRTI - 9.17. & $\begin{array}{l}\text { Utilidades pagadas en } \\
\text { Acciones y con préstamos } \\
\text { de la empresa, obteniendo } \\
\text { incremento de liquidez }\end{array}$ \\
\hline & $\begin{array}{l}\text { Deducción adicional del } 100 \% \text { de gastos } \\
\text { contraídos por las medianas empresas para: } \\
\text { 1)capacitación para la investigación, 2) mejora } \\
\text { de la productividad, 3) gastos contraídos en } \\
\text { viajes para la promoción comercial y el acceso } \\
\text { a mercados }\end{array}$ & LRTI - 10.17 & $\begin{array}{l}\text { La re inversión de utilidades } \\
\text { utilizadas en adquisición de } \\
\text { maquinaria para mejorar la } \\
\text { Producción }\end{array}$ \\
\hline \multirow{3}{*}{$\frac{\stackrel{0}{\frac{d}{0}}}{\frac{\mathrm{L}}{\mathrm{W}}}$} & $\begin{array}{l}\text { Deducción adicional del } 100 \% \text { durante } 5 \text { años } \\
\text { de las remuneraciones y beneficios sociales } \\
\text { por inversiones en zonas económicamente } \\
\text { deprimidas y de frontera. }\end{array}$ & LRTI - 10.9 & $\begin{array}{l}\text { Morona Santiago (MS) fue } \\
\text { declarada como zona } \\
\text { económicamente deprimida }\end{array}$ \\
\hline & $\begin{array}{l}\text { Deducción adicional del } 100 \% \text { de las } \\
\text { remuneraciones y beneficios sociales por } \\
\text { incremento neto de empleos. }\end{array}$ & LRTI - 10.9 & $\begin{array}{l}\text { observar las disposiciones } \\
\text { legales al respecto }\end{array}$ \\
\hline & $\begin{array}{l}\text { Deducción adicional del } 150 \% \text { correspondan a } \\
\text { remuneraciones y beneficios sociales sobre los } \\
\text { que se aporte al Instituto Ecuatoriano de } \\
\text { Seguridad Social, por pagos a adultos mayores } \\
\text { y migrantes retornados mayores de } 40 \text { años }\end{array}$ & LRTI - 10.9 & $\begin{array}{l}\text { Revisar puestos en los que } \\
\text { pudieran contratarse a } \\
\text { personal q cumpla el } \\
\text { requerimiento }\end{array}$ \\
\hline \multirow{3}{*}{$\begin{array}{l}\frac{1}{0} \\
\frac{0}{8} \\
\frac{0}{2} \\
0 \\
0 \\
0 \\
0\end{array}$} & $\begin{array}{l}\text { Reducción de } 10 \text { puntos en la tarifa aplicable } \\
\text { al monto de las utilidades reinvertidas en el } \\
\text { país para la mejora de la productividad. }\end{array}$ & $\begin{array}{l}\text { LRTI - 37, Ley } \\
\text { Reactivación } \\
\text { Económica } \\
\text { Art1.7 }\end{array}$ & $\begin{array}{l}\text { Reinvertir en maquinaria } \\
\text { que incremente la } \\
\text { producción Eje excavadora, } \\
\text { trituradora etc. }\end{array}$ \\
\hline & $\begin{array}{l}\text { Compensación de las pérdidas sufridas en el } \\
\text { ejercicio impositivo con las utilidades gravables } \\
\text { que obtuvieren dentro de los cinco períodos } \\
\text { impositivos siguientes. }\end{array}$ & LRTI - 11 & $\begin{array}{l}\text { Si ocurriera en años } \\
\text { posteriores }\end{array}$ \\
\hline & $\begin{array}{l}\text { Diferimiento por } 5 \text { años del pago del impuesto } \\
\text { y el anticipo por distribución de capital social } \\
\text { entre los trabajadores. }\end{array}$ & LRTI - 39.1 & $\begin{array}{l}\text { Analizar las situación } \\
\text { empresarial y esta posible } \\
\text { solución en apalancamiento }\end{array}$ \\
\hline
\end{tabular}

Fuente: Adaptado de LORTI (H. Congreso Nacional, 2007).

Las aplicaciones de los beneficios propuestos son ejemplificadas a continuación según la conveniencia de la empresa y la información proporcionada por la misma, más en los casos de 
compensación de pérdidas e intereses al personal por la compra de acciones, quedan planteadas para mediano plazo, dando a la administración una herramienta decisional que le permita prever escenarios a corto y mediano plazo en su manejo tributario administrativo.

Se propone utilizar el incremento neto de empleados, para lo cual se analizó el número de personas que cumplen con la condición de permanencia de seis meses trabajando en la empresa, de esta manera se puede ocupar el 100\% adicional de esos gastos como deducibles. Como la empresa se encuentra en una zona deprimida, y emplea a personas del mismo lugar, le permite acogerse a este incentivo por cinco años.

Tabla 6. Incremento neto de empleo

\begin{tabular}{|lr}
\hline \multicolumn{2}{|c}{ Macascorp S.A } \\
\hline \multicolumn{2}{|c|}{ Incremento neto de Empleo } \\
\hline Nómina de empleados 2018 & 77 \\
\hline Nómina de empleados $\mathbf{2 0 1 7}$ & 44 \\
\hline Diferencia en Nomina & 33 \\
\hline Empleados que cubren puestos bacantes & 16 \\
\hline Incremento de empleados & 17 \\
\hline No cumplen con condicionamientos & 12 \\
\hline Incremento Neto de empleados & 5 \\
\hline Remuneración aportada por empleado nuevo & 419,00 \\
\hline Meses laborados en el año & 7 \\
\hline Valor por Incremento Neto de empleo $\quad$ Verificación de valores cancelados & 14665,00 \\
\hline Sueldos año $2018 \quad \quad$ Incremento en Sueldos & 67550,67 \\
\hline Sueldos año 2017 & 50632,52 \\
\hline
\end{tabular}

Fuente: Roles y anexo de relación de dependencia de la empresa años 2017 - 2018 Reconocimiento de ingresos según lo que establece las NIIF 15. De la revisión de los estados financieros de la empresa se reconoce un pasivo, el cual tiene como fuente un anticipo recibido por parte del contratante, mismo que se vería como ya ejecutado según avances de obra, por tal razón en la siguiente tabla se describen las circunstancias que pudieran ser consideradas por la 
empresa en el reconocimiento de un nuevo ingreso y su impacto en los resultados del período fiscal.

Tabla 7. NIIF 15.- Construcción y Sector Inmobiliario

\begin{tabular}{|c|l|}
\hline Criterio & $\begin{array}{l}\text { El principio fundamental de la NIIF es que una entidad reconozca los ingresos que representen la } \\
\text { transferencia de bienes o servicios prometidos a los clientes en una cantidad ponderación que la } \\
\text { entidad estima tener derecho por el cambio de esos bienes y servicios }\end{array}$ \\
\hline Condición & $\begin{array}{l}\text { La empresa en sus estados financieros contempla un valor de } \$ 83584,63 \text { dolares } \\
\text { correspondientes a anticipo de obra pendiente de debengar, el mismo a a diciembre se pudo } \\
\text { haber realizado el reconocimiento como ingreso para la empresa }\end{array}$ \\
\hline Causa & $\begin{array}{l}\text { La no aplicación de la normativa contable en virtud de la falta de autorización de la entidad } \\
\text { contratante para que se proceda con el planillaje y factura correspondiente }\end{array}$ \\
\hline Efecto & Una sub estimación de los ingresos, y menores rendimientos financieros \\
\hline
\end{tabular}

Fuente: Elaboración propia

Reinversión de utilidades: La reinversión de utilidades es un beneficio tributario conocido por los empresarios, pero no muy utilizado ya que para su uso se debe realizar cálculos matemáticos según la fórmula específica entregada por el SRI en la LORTI y su reglamento, dicha fórmula limita el monto máximo que la empresa puede reinvertir de acuerdo con parámetros individuales como la reserva legal, base imponible, entre otros.

Tabla 8. Reinversión de utilidades actual 
ISSN: 2602-8506

Vol. 3, $\mathrm{N}^{\circ} 2.1 .$, p. 6-34, junio, 2019

\begin{tabular}{|c|c|c|}
\hline \multirow{2}{*}{ Fórmula } & \multicolumn{2}{|c|}{$\left(1-\%\right.$ RL) * UE ] - $\left\{[\% \text { IRO - (\% IRO * \% RL) }]^{*} \mathrm{BI}\right\}$} \\
\hline & \multicolumn{2}{|c|}{$1-(\%|R O-\%| R I)+\left[(\%|R O-\%| R I){ }^{*} R L\right]$} \\
\hline Nomenclatura & Descripción & 2018 \\
\hline $\begin{array}{l}\text { (\% RL) Reserva } \\
\text { legal }\end{array}$ & $\begin{array}{l}\text { Porcentaje de reserva legal } 0 \%, 5 \% \text {, o 10\% según } \\
\text { corresponda }\end{array}$ & $0 \%$ \\
\hline $\begin{array}{l}\text { (UE) Utilidad } \\
\text { efectiva }\end{array}$ & $\begin{array}{l}\text { Utilidad contable disminuida la participación de } \\
\text { trabajadores }\end{array}$ & $79.134,63$ \\
\hline $\begin{array}{l}\text { (\% IRO) Tarifa } \\
\text { original }\end{array}$ & Tarifa de impuesto a la renta, para el 2018 es el $25 \%$ & $25 \%$ \\
\hline $\begin{array}{l}\text { (\% IRI) Tarifa } \\
\text { reducida }\end{array}$ & Es igual a la tarifa original menos 10 puntos de incentivo. & $15 \%$ \\
\hline \multirow{2}{*}{$\begin{array}{c}\text { (BI) Base } \\
\text { imponible }\end{array}$} & Calculada conforme a disposiciones legales tributarias & \multirow{2}{*}{$67.865,11$} \\
\hline & Para el caso práctico UE + GND & \\
\hline \multicolumn{2}{|r|}{ Valor máximo a reinvertir } & $65.002,34$ \\
\hline IR reducido & Valor a re invertir $x$ el \%IRI & $9.750,35$ \\
\hline IR sin reducción & ( UE - Valor de re inversión) x \%IRO & 715,69 \\
\hline \multicolumn{2}{|r|}{ Total Impuesto Causado } & $10.466,04$ \\
\hline
\end{tabular}

Fuente: Estados financieros de la empresa

Evaluación de resultados: Para evaluar los resultados propuestos se plantea una conciliación tributaria en la cual se mide el impacto de la propuesta resumida en reconocimiento de un ingreso adicional conjuntamente con el aumento de los gastos deducibles y la disminución de los no deducibles y finalmente se concluye con la utilización de beneficios tributarios como el incremento neto de empleo y la disminución de la tasa impositiva por re inversión de las utilidades.

Además, se presenta a continuación un nuevo cálculo de la tasa efectiva o carga tributaria de la empresa propuesta, conjuntamente con un análisis del indicador Dupont y sus variaciones, verificando si el impacto de la planificación tributaria también afecta a los resultados de dicho indicador,

Tabla 9. Conciliación tributaria propuesta 
ISSN: 2602-8506

Vol. 3, N².1., p. 6-34, junio, 2019

\begin{tabular}{|c|c|c|c|}
\hline Concepto & Situación Actual & $\begin{array}{c}\text { Con Planificación } \\
\text { Tributaria }\end{array}$ & Referencia \\
\hline Ingresos & $1.062 .445,26$ & $1.146 .029,89$ & Tabla 7 \\
\hline Costos y Gastos & $1.037 .953,13$ & $1.052 .930,32$ & Tabla 4 \\
\hline Utilidad Contable & $24.492,13$ & \multicolumn{2}{|l|}{$93.099,57$} \\
\hline (-15\%) Participación Trabajadores & $3.673,82$ & $13.964,94$ & \\
\hline (=) Utilidad efectiva & $20.818,31$ & \multicolumn{2}{|l|}{$79.134,63$} \\
\hline Conciliación tributaria para cálculo de reinversión & & & \\
\hline (+) Utilidad Efectiva & $20.818,31$ & \multicolumn{2}{|l|}{$79.134,63$} \\
\hline (-) $100 \%$ Ganancia por venta de acciones & & & Tabla 5 \\
\hline (-) $100 \%$ Intereses préstamos empleados compra acciones & & \multicolumn{2}{|r|}{ Tabla 5} \\
\hline (+) Gastos no deducibles & $39.365,72$ & $3.395,48$ & \\
\hline \multicolumn{4}{|l|}{ (+) Participación trabajadores atribuibles rentas exentas } \\
\hline \multicolumn{4}{|l|}{ (-) Amortización perdidas tributarias de años anteriores } \\
\hline (=) Base imponible para el cálculo de la reinversión & $60.184,03$ & \multicolumn{2}{|l|}{$82.530,11$} \\
\hline (-) Deducción adicional por mejoras productividad & & & Tabla 5 \\
\hline (-) Deducción por incremento neto de empleados & & $14.665,00$ & Tabla 6 \\
\hline (-) Deducción trabajadores con discapacidad & & & Tabla 5 \\
\hline \multicolumn{4}{|c|}{ (-) Deducción adicional empresas constructoras o sectores deprimidos } \\
\hline Utilidad Imponible & $60.184,03$ & $67.865,11$ & \\
\hline (-) Utilidad a reinvertir y capitalizar (15\% impuesto a la renta) & & $65.002,34$ & Tabla 8 \\
\hline (=) Utilidad imponible (25\% impuesto a la renta) & & $2.862,77$ & Tabla 8 \\
\hline \multicolumn{4}{|l|}{ Impuesto a la Renta acogiéndose a reinversión y demás beneficios } \\
\hline Impuesto sobre el valor a reinvertir (15\%) & & $9.750,35$ & Tabla 8 \\
\hline Impuesto sobre el saldo de la utilidad imponible (25\% & $15.046,01$ & \multicolumn{2}{|l|}{715,69} \\
\hline (=) Impuesto causado & $15.046,01$ & $10.466,04$ & \\
\hline (=) Utilidad del Ejercicio & $5.772,30$ & \multicolumn{2}{|l|}{$57.399,07$} \\
\hline (-) Reserva Legal (10\%) & - & - & \\
\hline (=) Utilidad a reinvertir y capitalizar & - & \multicolumn{2}{|l|}{$57.399,07$} \\
\hline (-) Utilidad a reinvertir y capitalizar ( $15 \%$ Impuesto a la renta) & - & $55.251,99$ & \\
\hline (=) Utilidad a Distribuir & $5.772,30$ & \multicolumn{2}{|l|}{$2.147,08$} \\
\hline
\end{tabular}

Fuente: Adaptado del formulario 101 del SRI 
ISSN: 2602-8506

Vol. 3, N².1., p. 6-34, junio, 2019

www.visionariodigital.org

Si bien, la utilidad a distribuir en el panorama propuesto es menor en aproximadamente un cincuenta por ciento, el impuesto a pagar es menor y la empresa contaría con un capital a reinvertir de alrededor de cincuenta mil dólares, que a más de optimizar impuestos mejora sus condiciones financieras.

Tabla 10. Comparación principales cuentas contables y TE

\begin{tabular}{|lrrrr}
\hline \multicolumn{1}{c}{ Rubro } & \multicolumn{1}{c}{ Actual } & \multicolumn{1}{c}{ Propuesto } & Variación \\
\hline Activos Totales & $1.326 .091,34$ & $1.311 .114,15$ & - & $14.977,19$ \\
\hline Pasivos Totales & $1.006 .862,79$ & $923.278,16$ & - & $83.584,63$ \\
\hline Patrimonio & $319.228,55$ & $387.835,99$ & $68.607,44$ \\
\hline Ventas & $1.062 .445,26$ & $1.146 .029,89$ & $83.584,63$ \\
\hline Costo de Ventas & $1.002 .898,05$ & $1.017 .875,24$ & $14.977,19$ \\
\hline Gastos & $35.055,08$ & $35.055,08$ & - \\
\hline Utilidad & $24.492,13$ & $93.099,57$ & $68.607,44$ \\
\hline 15\% Trabajadores & $3.673,82$ & $13.964,94$ & $10.291,12$ \\
\hline Utilidad Efectiva & $20.818,31$ & $79.134,63$ & $58.316,32$ \\
\hline Impuesto Causado & $15.046,01$ & $10.466,04$ & - \\
\hline Gastos No Deducibles & $39.365,72$ & $3.395,48$ & - & $35.970,97$ \\
\hline \multicolumn{1}{c}{ TE } & & & \\
\hline \multicolumn{1}{c}{} & $\mathbf{1 , 4 2 \%}$ & $\mathbf{0 , 9 1 \%}$ & \\
\hline
\end{tabular}

Fuente: Elaboración propia

La utilidad efectiva en el escenario propuesto se incrementa tres veces de su panorama actual, mientras que el impuesto causado disminuye en un cuarenta por ciento, esto debido principalmente a la disminución de los gastos no deducibles y el uso de beneficios tributarios.

Tabla 11. Índice Dupont 
ISSN: 2602-8506

Vol. 3, N².1., p. 6-34, junio, 2019

\begin{tabular}{|c|c|c|c|c|c|}
\hline \multicolumn{6}{|c|}{ Cálculo Indice Dupont } \\
\hline \multirow{4}{*}{$\stackrel{\infty}{\stackrel{\sim}{\circ}}$} & Margen Neto de Utilidad & Utilidad neta/ Ventas & $24.492,13$ & $1.062 .445,26$ & 0,02 \\
\hline & Rotación de activos totales & Ventas/ Activo Total & $1.062 .445,26$ & $1.326 .091,34$ & 0,80 \\
\hline & Apalancamiento Financiero & Activos / Patrimonio & $1.326 .091,34$ & $319.228,55$ & 4,15 \\
\hline & Sistema Dupont & \multicolumn{2}{|c|}{ Margen $\mathrm{N} *$ Rotacion Act* Ap Financierp } & & 0,08 \\
\hline \multirow{4}{*}{$\begin{array}{l}\llcorner \\
\infty \\
\infty \\
\stackrel{-1}{0}\end{array}$} & Margen Neto de Utilidad & Utilidad neta/ Ventas & $93.099,57$ & $1.146 .029,89$ & 0,08 \\
\hline & Rotación de activos totales & Ventas/ Activo Total & $1.146 .029,89$ & 1.311.114,15 & 0,87 \\
\hline & Apalancamiento Financiero & Activos / Patrimonio & 1.311.114,15 & $387.835,99$ & 3,38 \\
\hline & Sistema Dupont & \multicolumn{2}{|c|}{ Margen $\mathrm{N} *$ Rotacion Act* Ap Financierp } & & 0,24 \\
\hline
\end{tabular}

Fuente: Elaboración propia

Un sustancial incremento de índice Dupont propuesto en un trecientos por ciento a comparación de la inicial, demuestra una mejor gestión integral de los recursos de la empresa, tal aumento es causado por un lado por el incremento exponencial del margen operacional, mientras que por otro una reducción marginal del apalancamiento financiero y finalmente manteniéndose la rotación de los activos empresariales. Por lo tanto, la PT nos ayuda a optimizar los recursos de la empresa no solamente desde la perspectiva tributaria sino también financiera.

\section{Conclusiones:}

- Ante una mayor presión fiscal que ejerza el Estado, las empresas buscarán estrategias mediante las cuales se reduzcan los impuestos a pagar, estas estrategias estarán apegadas al marco legal o no, por tanto, es responsabilidad del Estado ejercer actividades de control para fortalecer la recaudación, mientras que las empresas deben propender usar estrategias técnicas y legales para obtener sus objetivos sin riego a futuro.

- En el Ecuador, la presión fiscal en los últimos años ha tenido una evolución creciente, y los distintos controles llevados a cabo por la administración tributaria afectaron sustancialmente los resultados de las compañías del sector constructor. Por lo que, toda 
ISSN: 2602-8506

Vol. 3, $\mathrm{N}^{\circ} 2.1 .$, p. 6-34, junio, 2019

empresa que desee ser competitiva debe controlar sus recursos, siendo la planificación tributaria una herramienta financiera o gerencial que coadyuva a la toma de decisiones tanto en la utilización óptima de los recursos como en la consecución de los objetivos empresariales planteados.

- Una planificación tributaria no propende solamente la disminución de los impuestos a pagar, también busca su optimización; no sólo el pago de menos impuestos beneficia a la empresa, el manejo correcto de los impuestos pagados o a pagar, sumado al uso de los distintos beneficios tributarios y un correcto manejo administrativo conjugado en una planificación tributaria ajustada a la empresa en estudio puede ser mayormente beneficiosa; esta conclusión claramente demostrada mediante el indicador Dupont aplicado en estudio, que para el caso práctico duplico el indicador con la propuesta planteada.

- La presentación de esta planificación tributaria a la empresa Constructora y Planificación Macas Macascorp S.A., de la ciudad de Macas, otorgará a los altos directivos la posibilidad de tomar mejores decisiones en cuanto a la gestión tributaria, ya que la misma permite medir su nivel de riesgo tributario, optimizar sus recursos y elevar su competitividad.

\section{Bibliografía}

Servicio de Rentas Internas. (2015). Plan Estrategico Institucional 2016-2019. Quito: Servicio de Rentas Internas.

Acevedo, S. (1998). Planificación Tributaria y Administración de Capital de Trabajo. Colombia: Norma.

Amorós, N. (1965). La elusión y la evasión tributaria. Madrid: Revista de Derecho Financiero y Hacienda Pública.

Cámara, L. (2005). Planeación Estrategica. Madrid: CIDEAL.

Cazau, P. (2006). Introducción a la Investigación en Ciencias Sociales 3ra Edición. Buenos Aires. 
Vol. 3, N².1., p. 6-34, junio, 2019

Crespo, A. (2016). La Presión Fiscal en el Ecuador: Análisis de la Carga Tributaria (Tesis Maestría). Guayaquil: Instituto de Altos Estudios Nacionales.

Cusgüen, E. (1998). Planificación del tributo, impuestos. Madrid: Colección Europa.

Delgado, G. (2010). Conceptos y metodología de la investigación histórica. Revista Cubana de Salud Pública, 9-18.

Dirección Nacional de Investigación y Estudios. (2016). Breve análisis societario del sector de la construcción del Ecuador 2014-2015. Quito: Superintendencia de Compañías, valores y seguros.

Dupont. (17 de 03 de 2018). Dupont.com. Obtenido de Dupont.com: http://www2. dupont.com/Phoenix_Heritage/en_US/1918_detail.html

Galárraga, A. (2002). Fundamentos de la Planificación Tributaria. Carácas: Editorial Jurídica Venezolana.

García, O. (2014). Fórmula Du Pont y su rentabilidad, vista desde la óptica administrativa. Inquietud Empresarial, 89-113.

Guizar, R. (2013). Desarrolo organizacional, principios y aplicaciones. México D.F.: Mc Graw Hill.

H. Congreso Nacional, E. (30 de 11 de 2007). LORTI. LORTI. Quito, Pichincha, Ecuador: RO. 223.

Hidalgo, A. (20 de 12 de 2009). https://www.urbe.edu. Obtenido de Aspectos generales de la planificación tributaria en Venezuela:

https://www.urbe.edu/UDWLibrary/ArticulosAdvance.do?operator=EMPTY\&tag=100\& word=Hidalgo\%20de\%20Camba,\%20Alejandra

Instituto de Desarrollo Industrial, Tecnológico y de Servicio. (2009). Informe Final: Sector Construcción, Parte. Argentina: inet. 
ISSN: 2602-8506

Vol. 3, $\mathrm{N}^{\circ} 2.1 .$, p. 6-34, junio, 2019

Instituto Nacional de Estadistica y Censos INEC. (10 de Diciembre de 2012). La Industria de la Construcción es el mayor empleador del mundo. Obtenido de ecuadorencifras.gob.ec: http://www.ecuadorencifras.gob.ec/wp-content/descargas/Infoconomia/info10.pdf

Llanos, N. (05 de 11 de 2018). ACADEMIA. Obtenido de ACADEMIA.EDU: http://www.academia.edu/5075869/CLASES_Y_TIPOS_DE_INVESTIGACION_Y_SU S_CARACTERISTICAS

López, K. (2017). La planificación tributaria como herramienta financiera para la toma de decisiones en el sector inmobiliarioa. Quito.

Medizabal, L. (2014). Análisis Jurídico del la Evación y Elución Fiscal en Guatemala (Tesis de Posgrado). Guatemala: Universidad Rafael Landívar.

Ministerio de Justicia, Derechos Humanos y Cultos. (02 de 10 de 2014). Código Orgánico Integral Penal. Código Orgánico Integral Penal. Quito, Pichincha, Ecuador: RO 180.

Osar, C., \& Ramos, N. (20 de 12 de 2017). El portal fiscal y el control tributario. Obtenido de El portal fiscal y el control tributario: www.nerlassociados.com

Parra, A. (2007). Planeación Tributaria y Organización Empresarial. Colombia: LEGIS.

Ramirez, A. (2003). Metodología de la investigación científica. Bogotá: Pontificia Universidad Javeriana.

Rivas, N., \& Vergara, S. (2002). Planificación Tributaria. Santiago de Chile: Magril.

Ruiz, J. (1998). El Fraude de Ley y Otros Supuestos de Elusión Fiscal. Valencia: CISS S.A.

Sánchez, L., \& Hablich, F. (2018). Contribución de la planificación. Espacios, 15.

SRI. (15 de 03 de 2018). Visores Tributarios. Obtenido de Visores Tributarios: http://visoresestadisticos.sri.gob.ec/qap/single/?appid=73ae68ff-7352-40cb-8b1a053e4ec0838a\&sheet=6ac5c195-1f61-477a-8f4f-bb6801e4b73f\&lang=esES\&opt=currsel 
ISSN: 2602-8506

www.visionariodigital.org

Vol. 3, N².1., p. 6-34, junio, 2019

Tipker, K. (2002). Moral Tributaria del Estado y de los contribuyentes. Madrid: Marcial Pons.

Villafuerte, D. (2006). Manual Metodologico para el Investigador Cientifico. Parú: U.N.S.A.

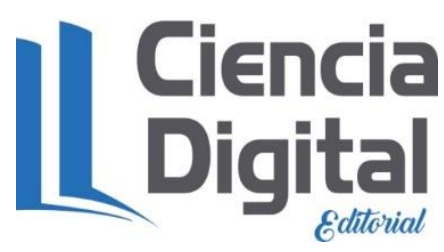




\section{PARA CITAR EL ARTÍCULO INDEXADO.}

Mena Trujillo, R., Narváez Zurita, C., \& Erazo Álvarez, J. (2019). La planificación tributaria como herramienta financiera para la optimización de los recursos empresariales. Visionario Digital, 3(2.1.), 6-34. https://doi.org/10.33262/visionariodigital.v3i2.1.540

\section{LCiencia}

El artículo que se publica es de exclusiva responsabilidad de los autores y no necesariamente reflejan el pensamiento de la Revista Visionario Digital.

El artículo queda en propiedad de la revista y, por tanto, su publicación parcial y/o total en otro medio tiene que ser autorizado por el director de la Revista Visionario Digital.
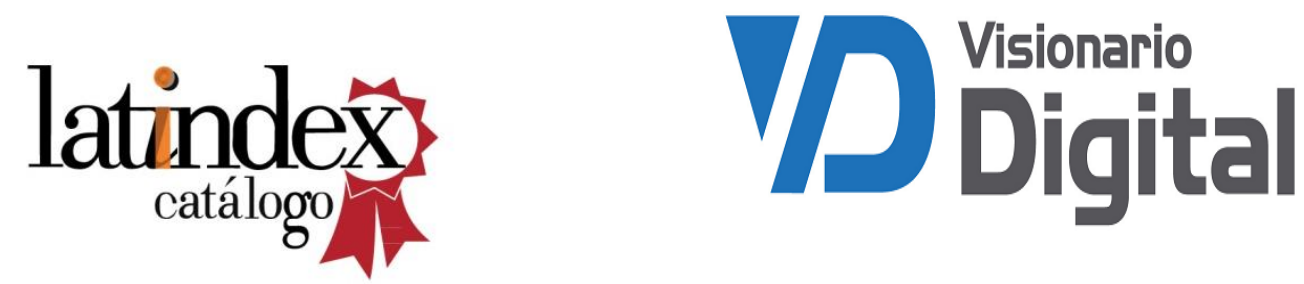